\title{
Maternal Mortality in the UK: An Update
}

Bryn Kemp, MRCOG DPhil

*Marian Knight, MA MBChB MPH DPhil FFPH FRCPE

National Perinatal Epidemiology Unit

University of Oxford

Old Rd Campus

Oxford

OX3 7LF

${ }^{*}$ Corresponding author

Email: marian.knight@npeu.ox.ac.uk

Tel: $+44-1865-289727$ 


\section{Abstract}

The latest report of the United Kingdom Confidential Enquiry into Maternal Mortality, conducted by the MBRRACE-UK collaboration, was published in December 2014. The report has moved from triennial to annual publication with a chapter on each specific cause of maternal death included once every three years. In 2010-12, overall maternal mortality fell to 10.1 per 100,000 maternities; a $27 \%$ decrease compared to $2003-5$. Whilst the maternal mortality rate from genital tract sepsis more than halved from its 20-year high in 2006-2008, sepsis per-se accounted for almost $25 \%$ of deaths. One in 11 of all deaths were associated with sepsis related to influenza, the majority 2009/A H1N1 influenza, which, in the presence of an effective vaccine, were largely preventable. The benefits of influenza vaccination should be promoted and women offered vaccination at any stage of pregnancy. Thrombosis was the leading cause of direct death, highlighting the ongoing importance of thromboprophylaxis.

\section{Key words}

Maternal mortality; sepsis; influenza, haemorrhage, epilepsy 


\section{Introduction}

The UK Confidential Enquiry into Maternal Deaths recognises that every maternal death is a tragedy; to families, to the staff involved, and to the wider communities left behind. Since the first report in 1952, the maternal mortality rate in the UK has fallen from approximately 90 per 100,000 to 10 per 100,000 maternities. The Enquiry is now conducted by the MBRRACEUK collaboration and the latest report includes for the first time review of the care of women from Ireland. In addition, there is a move to publication of annual rather than triennial reports, with a chapter on each specific cause of maternal death included once every three years, alongside topic specific reviews of episodes of maternal morbidity. In the 2014 report, chapters reviewed morbidity and mortality relating to maternal sepsis along with deaths related to haemorrhage, amniotic fluid embolism, anaesthesia-related deaths and deaths from neurological and other indirect causes.

Between 2009-2012, 357 women died during, or within six weeks of the end of their pregnancy in the UK. Thirty-six deaths were classified as coincidental, thus there were 321 maternal deaths in 2009-12. The maternal mortality rate of 10.1 per 100,000 maternities in 2010-12 represents a statistically significant reduction of $27 \%$ since $2003-5$ (relative risk [RR] $0.73,95 \%$ confidence interval $[\mathrm{Cl}]$ 0.61-0.89). Almost all of this observed reduction is due to a decline in direct (obstetric) deaths, which fell by $48 \%$ over this period ( $\mathrm{RR} 0.52,95 \% \mathrm{Cl} 0.39$ 0.69). The mortality rate from indirect deaths has, however, remained unchanged for more than 10 years ( $\mathrm{RR} 0.90,95 \% \mathrm{Cl} 0.72-1.11$ ). This observed reduction in the maternal mortality rate has occurred on the background of an increase in the number of maternities, and alongside rising levels of obesity, advancing maternal age, and an increasing proportion of births amongst women born outside the UK; all of which serve to increase the risk of maternal death. 


\section{The Women Who Died}

Amongst the 321 women who died, $30 \%$ were still pregnant at the time of death. Of these, one third were under 20 weeks' gestation. Amongst all women: $74 \%$ had pre-existing medical complications; $27 \%$ were obese, an independent risk factor for maternal death; almost 1 in 10 received no antenatal care, and $25 \%$ received care below the minimum standard defined by the National Institute of Health and Clinical Excellence (NICE).

\section{Direct and indirect deaths: Key facts}

Whilst the maternal mortality rate from genital tract sepsis more than halved from its 20-year high in 2006-2008 (RR 0.44, 95\% Cl 0.22-0.87), sepsis from all causes accounted for almost $25 \%$ of deaths. In particular, one in 11 of all deaths were associated with sepsis related to influenza, the majority 2009/A H1N1 influenza, which, in the presence of an effective vaccine, were largely preventable. The benefits of influenza vaccination should be promoted and women offered vaccination at any stage of pregnancy. Thrombosis was the leading cause of direct death, highlighting the ongoing importance of thromboprophylaxis.

\section{General Messages for Care}

\section{1) Clinical observations}

All women showing any signs or symptoms of ill health require a full set of basic observations, including heart rate, temperature, respiratory rate and blood pressure; the results should be documented and acted upon. The reviewers frequently found that the respiratory rate was not measured. This is as important a physiological measure as the pulse rate, blood pressure and temperature. Sepsis causes an increased respiratory rate to meet an increased oxygen demand of the tissues, as well as in possible compensation for metabolic acidosis or due to 
the presence of acute respiratory distress syndrome (ARDS). The threshold for the upper limit of normal in pregnancy is 20 breaths per minute..

\section{2) Avoid referral delays}

Delays in escalating the care of complex cases contributed to poor outcomes in a number of cases, and it is important to be aware of 'red flags' for escalating care such as those highlighted in relation to sepsis below. Junior medical and midwifery staff should not hesitate to seek advice from senior colleagues, particularly at night and weekends, when on-site cover may be limited. Consultant to consultant referral should be undertaken in cases where specialist advice is required to minimise delays when assessing and treating women.

\section{3) Clinical service configuration}

Almost three quarters of women who died from both direct and indirect causes had preexisting medical problems, including a wide range of different conditions across all body systems. Such women represent a high-risk group and need to receive the appropriate individualised multidisciplinary evidence-based care pre-pregnancy, during pregnancy and after delivery.

\section{Topic Specific Messages}

\section{1) "Think sepsis"}

Sepsis refers to an infection with coexisting features of the systemic inflammatory response (SIRS) (see Box). Eighty-three women died of sepsis related causes (maternal mortality rate 2.04 per 100,000 maternities).

Whilst there were examples of excellent care being provided to women, delays in the diagnosis and management of sepsis was a recurring theme. Repeated non-specific presentations to non-obstetric services, may be indicative of sepsis and women should be evaluated for 'red flag' sepsis (see Box). Where sepsis is suspected, a sepsis care-bundle should be initiated with urgency. National guidance requires that obstetric units have a 
protocol in place outlining a locally agreed sepsis bundle, along with relevant clinical governance and audit pathways necessary to the monitor implementation and efficacy of sepsis care pathways.

Box 3.1: SIRS screening and evaluation for Red Flag Sepsis (UK Sepsis Trust Primary Care Toolkit 2014)

a. Screening for SIRS

SIRS is confirmed if ANY TWO of the following are present:

Immediate

- New onset of Confusion or Altered Mental State

- Temperature $>38.3^{\circ} \mathrm{C}$ or $<36^{\circ} \mathrm{C}$

- Heart Rate $>90$ beats per minute*

- Respiratory Rate (counted over 60 seconds) >20 breaths per minute

Point of Care Test (commonly available)

- Blood Glucose $>7.7 \mathrm{mmol} / \mathrm{L}$ in the absence of known diabetes

Point of Care Test (not yet widely available)

- White cell count $>12$ or $<4 \times 10^{9} / \mathrm{L}$

b. Evaluation for Red Flag Sepsis

Act immediately if ANY ONE of the following are present:

- Systolic BP $<90 \mathrm{mmHg}$ (or $>40 \mathrm{mmHg}$ fall from baseline)

- Heart rate $>130$ per minute

- Oxygen saturations $<91 \%$

- Respiratory rate $>25$ per minute

- Responds only to voice or pain/ unresponsive

Point of Care test (not yet widely available)

- Lactate $>2.0 \mathrm{mmol} / \mathrm{L}$

* Note the guidelines are not specific for pregnancy and these observations should be interpreted in the context of the normal physiology for the pregnant woman. RCOG guidance suggests using a threshold of 100 beats per minute in pregnancy (RCOG Green-top Guideline 64a).

Reproduced with permission from Saving Lives, Improving Mothers' Care 2014 


\section{2) Prevent and treat haemorrhage}

Obstetric haemorrhage accounted for $10 \%$ of direct maternal deaths in the UK. Seventeen women died (maternal mortality rate 0.49 per 100,000 maternities) with an estimated case fatality rate for massive obstetric haemorrhage of 1 per 1200 episodes. Two deaths followed placental abruption, one was in association with placenta praevia, seven deaths followed uterine atony and seven following genital tract trauma including uterine rupture. All deaths were related to peri-delivery haemorrhage.

During acute haemorrhage, involvement from senior obstetric, anaesthetic and midwifery staff is essential and concerns should be escalated if a woman deteriorates. Resuscitation should not be guided by single point estimates of haemoglobin in the acute phase of haemorrhage, which can mislead assessments of the severity of blood loss, but by estimated blood loss as well as clinical symptoms and signs. Up to four units of fresh frozen plasma and ten units of cryoprecipitate may be given empirically in cases of severe bleeding, alongside either O-negative or cross-matched blood while awaiting the results of coagulation studies. Rapid infusers and fluid warmers should be readily available, and early recourse to hysterectomy is essential when more conservative interventions prove ineffective.

\section{3) Care for women with Amniotic Fluid Embolism}

Amniotic fluid embolism (AFE) has a case fatality rate of $11-61 \%$ and affects 1 in 50,000 pregnant women. Between 2009-12, 11 women died from AFE (maternal mortality rate 0.33 per 100,000 maternities); messages for care were similar to those for women with haemorrhage. In extremis, for women in cardiac arrest, perimortem caesarean section should be carried out within five minutes of diagnosis; remembering that it is performed for maternal reasons alone, with no need to confirm fetal viability beforehand. 


\section{4) Women with epilepsy}

Epilepsy affects $1 \%$ of the population and was responsible for 14 maternal deaths (maternal mortality rate 0.40 per 100,000 maternities), more than any single direct cause of death with the exception of thrombosis; and unchanged from the previous triennium. Pregnant women with epilepsy should have their treatment optimised before conception and should have access to joint obstetric and neurological assessment. Multi-agency guidelines for the care of pregnant women with epilepsy are urgently needed; an RCOG national guideline is currently under development.

\section{5) Women with stroke and sub-arachnoid haemorrhage}

Between 2009-2012, 26 women died with intracranial haemorrhage (maternal mortality rate 0.75 per 100,000 maternities). Women presenting with headache should have a full neurological examination, including fundoscopy and an assessment for the presence of neck stiffness. Where stroke is subsequently diagnosed, care should be within a hyperacute stroke unit, and pregnancy, caesarean delivery or the immediate postpartum state should not be considered absolute contraindications to treatment with thrombolysis.

\section{6) Other medical complications}

Amongst women who died from other medical complications, reviewers identified a number of common themes. These included a general absence of preconception care or treatment optimisation for women with chronic disease. There was also a tendency for women to alter treatment plans and to stop medication when pregnant. It was considered essential that women requiring input from multiple specialties should have a lead clinician nominated to be responsible for joined-up care across teams.

\section{Conclusion}


The latest UK Confidential Enquiry into Maternal Deaths and Morbidity clearly identified improvements in care, particularly in relation to the increasingly complex needs of pregnant women with medical co-morbidities in the UK. As clinical services continue to be restructured, and as clinical staff become increasingly sub-specialised, maternity care providers should focus on developing multidisciplinary care pathways including pre-pregnancy, during pregnancy and post-delivery, with an emphasis on early involvement of appropriate senior clinical staff.

\section{Conflicts of interest}

None to declare

\section{Funding}

The Maternal, Newborn and Infant Clinical Outcome Review programme, delivered by MBRRACE-UK, is commissioned by the Healthcare Quality Improvement Partnership (HQIP) on behalf of NHS England, NHS Wales, the Health and Social Care division of the Scottish government, the Northern Ireland Department of Health, Social Services and Public Safety (DHSSPS), the States of Jersey, Guernsey, and the Isle of Man.

BK is funded by an NIHR Academic Clinical Lectureship, MK is funded by an NIHR Research Professorship. The views expressed are those of the author(s) and not necessarily those of the NHS, the NIHR or the Department of Health. 


\section{Further reading}

Heslehurst, N., J. Rankin, J. R. Wilkinson and C. D. Summerbell (2010). "A nationally representative study of maternal obesity in England, UK: trends in incidence and demographic inequalities in 619323 births, 1989-2007." Int J Obes (Lond) 34(3): 420-428.

Knight M, Kenyon S, Brocklehurst P, Neilson J, Shakespeare J, Kurinczuk JJ (Eds) on behalf of MBRRACE-UK (2014). Saving Lives, Improving Mothers' Care - Lessons learned to inform future maternity care from the UK and Ireland Confidential Enquiries into Maternal Deaths and Morbidity 2009-12. Oxford: National Perinatal Epidemiology Unit, University of Oxford.

Nair, M., J. J. Kurinczuk, P. Brocklehurst, S. Sellers, G. Lewis and M. Knight (2015). "Factors associated with maternal death from direct pregnancy complications: a UK national casecontrol study." BJOG 122(5): 653-662.

National Institute for Health and Care Excellence. (2008). "CG62: Antenatal care." Retrieved 15/04/2015, from http://www.nice.org.uk/guidance/cg62.

Royal College of Obstetricians and Gynaecologists. (2011). "Green-top Guideline 52: Postpartum Haemorrhage, Prevention and Management." from http://www.rcog.org.uk/womens-health/clinical-guidance/prevention-and-managementpostpartum-haemorrhage-green-top-52.

Royal College of Obstetricians and Gynaecologists. (2012). "Green-top Guideline No. 64a. Bacterial Sepsis in Pregnancy." from http://www.rcog.org.uk/files/rcogcorp/25.4.12GTG64a.pdf.

Royal College of Obstetricians and Gynaecologists. (2012). "Green-top Guideline No. 64b. Bacterial Sepsis following Pregnancy." from http://www.rcog.org.uk/files/rcogcorp/25.4.12GTG64a.pdf.

Royal College of Obstetricians and Gynaecologists. (2015). "Green-top Guideline 37a: Reducing the Risk of Thrombosis and Embolism During Pregnancy and the Puerperium." from http://www.rcog.org.uk/womens-health/clinical-guidance/reducing-risk-of-thrombosisgreentop37a.

United Kingdom Blood Services (2013). Handbook of Transfusion Medicine - 5th Edition.

UK Sepsis Trust. Clinical Tools. 2013. http://sepsistrust.org/info-for-professionals/clinicaltools/ (accessed 02/07/14). 
